\title{
Sri Lanka : de la lutte contre le terrorisme à la catastrophe humanitaire
}

\author{
Delon Madavan
}

\section{OpenEdition}

\section{Journals}

\section{Édition électronique}

URL : http://journals.openedition.org/echogeo/11130

DOI : 10.4000/echogeo. 11130

ISSN : 1963-1197

\section{Éditeur}

Pôle de recherche pour l'organisation et la diffusion de l'information géographique (CNRS UMR 8586)

\section{Référence électronique}

Delon Madavan, «Sri Lanka : de la lutte contre le terrorisme à la catastrophe humanitaire », EchoGéo [En ligne], Sur le Vif, mis en ligne le 24 avril 2009, consulté le 30 avril 2019. URL : http:// journals.openedition.org/echogeo/11130; DOI : 10.4000/echogeo.11130

Ce document a été généré automatiquement le 30 avril 2019.

\section{(c) (i) (9)}

EchoGéo est mis à disposition selon les termes de la licence Creative Commons Attribution - Pas d'Utilisation Commerciale - Pas de Modification 4.0 International 


\title{
Sri Lanka: de la lutte contre le terrorisme à la catastrophe humanitaire
}

\author{
Delon Madavan
}

1 Depuis son retrait unilatéral du cessez-le-feu, en janvier 2008, le président Rajapakse a lancé, au nom de la lutte contre le terrorisme ${ }^{1}$ et de la restauration de l'intégrité territoriale du pays, une impressionnante campagne militaire contre les Tigres de Libération de l'Eelam Tamoul (LTTE) et son projet de créer un Etat indépendant pour la minorité tamoule. Ainsi, l'armée sri lankaise, qui s'était déjà emparée des possessions orientales des Tigres en 2007, a réussi à reconquérir la quasi-totalité de la région de Vanni, dans le nord de l'île, faisant tomber coup sur coup les villes de Kilinochchi et Mullaitivu. L'armée sri lankaise a ainsi réussi à cantonner les rebelles en déroute sur une portion de $13 \mathrm{~m}^{2}$ dans la région de Puthukkudiyirupu. Mais ces victoires militaires, que le gouvernement célèbre comme une victoire contre le terrorisme, sont surtout à l'origine d'une importante catastrophe humanitaire. En effet, plusieurs dizaines de milliers de civils tamouls (20 000 selon le gouvernement et 50000 selon le comité International de la Croix-Rouge) se retrouvent encore coincés entre les Tigres (surnom donné aux combattants LTTE) et l'armée. Plusieurs dizaines de milliers de déplacés tamouls, qui ont réussi à fuir la zone des combats, se retrouvent enfermés dans des camps de détention, alors que dans le même temps, la différence de perception du conflit entre Tamouls et Cingalais creuse le fossé entre les deux communautés.

\section{Dans la zone des combats}

2 Des dizaines de milliers de civils se retrouvent encore piégés sur une bande côtière de $13 \mathrm{~km}^{2}$ entre l'armée et les rebelles. La détérioration des conditions de vie et la violation quotidienne du droit international humanitaire protégeant les civils en temps de guerre par les belligérants sont à l'origine d'une véritable catastrophe humanitaire.

3 Le bombardement intensif pratiqué par l'armée sur des zones densément peuplées par des civils laisse entrevoir la faible considération du gouvernement pour ces déplacés qui 
ne sont plus considérés comme citoyens sri lankais mais comme terroristes ou sympathisants du LTTE. Depuis trois mois, l'armée bombarde et procède à des tirs de mortier à l'intérieur et à proximité d'une zone qu'elle avait pourtant unilatéralement déclarée comme zone de sûreté. Les écoles, édifices religieux, les camps de réfugiés ainsi que des hôpitaux ont été pris pour cible à de multiples reprises par l'armée. Rien que dans les deux premiers mois de l'année, cette opération militaire a coûté la vie à plus de 2000 personnes, fait plus de 5000 blessés et beaucoup parmi les survivants sont gagnés par la folie.

4 Ces déplacés tamouls, qui ont suivi de gré ou de force les Tigres lors de leurs différentes retraites, doivent d'autre part faire face à la pression du mouvement séparatiste qui refuse de les laisser fuir la zone de combat et n'hésite pas à ouvrir le feu sur ceux qui tentent de le faire. Pour faire face aux pertes qu'il a subies, le LTTE embrigade de force les personnes valides et les enfants et n'hésite pas à utiliser la population civile comme bouclier humain, en se mélangeant à celle-ci et en installant son armement à proximité d'elle.

5 La surpopulation, le manque d'eau, de nourriture, de soins et d'abris empire la souffrance des civils. La détérioration de la situation est telle que des porte-parole des Nations Unies ont rapporté des cas de mort dus à la faim ou à des maladies telles que la pneumonie ou la méningite. Cette situation est aggravée par la décision du gouvernement d'interdire aux agences non gouvernementales l'accès aux zones de combat. Ainsi, seul le Comité International de la Croix-Rouge est présent pour évacuer les blessés par ferry à Trincomalee alors que des organisations, comme Médecins Sans Frontières, disposent sur place du personnel et des équipements nécessaires pour soutenir l'effort de la CroixRouge. Des dizaines de milliers de civils de tous âges sont ainsi abandonnés à leur sort par le gouvernement qui préfère condamner des innocents plutôt que de risquer de laisser entrer des témoins gênants.

\section{Les camps de détention en zones gouvernementales}

Plus de 36000 déplacés, qui ont réussi à échapper à la zone de combat, sont placés par les autorités sri lankaises dans des camps de détention (nommés welfare centers). Ces camps, entourés de barbelés et placés sous l'autorité directe de l'armée, doivent officiellement permettre de filtrer les combattants LTTE des civils. Les Tamouls y sont maltraités et doivent supporter la pression psychologique des militaires qui les suspectent d'appartenir aux LTTE (Libération du 12 février 2009). La nature militaire de ces camps, la privation du droit de circuler de ces personnes, l'accès limité à leurs proches et aux organisations humanitaires, ainsi que la durée indéterminée de leur présence dans ces camps constituent de sévères violations du droit international. En agissant ainsi, le gouvernement donne davantage l'impression de considérer ces réfugiés comme des prisonniers de guerre que comme des citoyens que l'on essaie de réintégrer à la nation. Autre point inquiétant, le projet envisagé par le gouvernement de garder dans des camps, pendant trois ans, l'ensemble des Tamouls de la région de Wanni, soit environ 200000 personnes, sous prétexte de les réhabiliter. Si les protestations de l'agence pour les réfugiés de l'ONU ont fait reculer le gouvernement, ce dernier n'exclut pas la possibilité de garder ces déplacés durant un an et d'en laisser partir $80 \%$ à l'issue de cette période. Ce projet reste comme le précédent intolérable et en violation avec la réglementation internationale sur les réfugiés déplacés à l'intérieur d'un pays. 


\section{Perception du conflit par les Cingalais et les Tamouls}

7 La propagande gouvernementale, qui n'a rien à envier à celle des Tigres, a pris grand soin de présenter la campagne militaire comme une lutte contre le terrorisme et les velléités séparatistes tamoules. Le contrôle des médias est tel qu'il est difficile d'entendre des voix s'élever contre cette guerre ou s'alarmer sur le sort des civils tamouls. D'ailleurs, le gouvernement prend grand soin d'attribuer la perte des civils tamouls aux seuls Tigres et affirme n'avoir éliminé que des combattants LTTE. La glorification du président Rajapakse et de son armée est telle que l'on assiste tout simplement à la négation des atrocités commises à l'encontre des civils dans le nord. De façon générale, le sort des Tamouls suscite peu de compassion chez la communauté majoritaire de l'île. Au contraire, la perspective d'en finir avec la guerre civile, sans à avoir accordé la moindre concession, satisfait les ultranationalistes cingalais qui voient là une façon de régler une fois pour toute, par la force, le problème tamoul à Sri Lanka. Les quelques défenseurs d'une solution négociée, comme le National Peace Council, sont marginalisés et considérés comme pro-Tigres.

8 La situation dans le nord préoccupe fortement les Tamouls d'origine jaffnaise ${ }^{2}$ à Colombo. Ces derniers ont souvent eux-mêmes vécu dans la région des Vanni en tant que personnes déplacées entre les années 1995 et 2002 et plusieurs ont encore des membres de leur famille ou des amis dans la zone de combat ou dans les camps de détention. Pour eux, la politique militaire et l'impunité dont bénéficie l'armée conforte leur idée que le gouvernement en place n'accorde aucune importance à leur vie et profite du facteur terroriste pour tuer des innocents. Les Tamouls ne se sentent plus considérés comme citoyens à Sri Lanka et ont peur d'être oubliés dans leur agonie par le reste du monde.

9 L'émotion suscitée par la catastrophe humanitaire en cours ne cesse de croître chez les Tamouls d'Inde et de la diaspora. Des voix s'élèvent pour dénoncer le génocide dont sont victimes les Tamouls et préconisent la création d'un tribunal pénal international pour Sri Lanka, comparant le massacre de Mullaitivu à celui de Srebrenica dans l'ex-Yougoslavie ( Tamoulobs du 12 avril 2009). Que l'on soit ou non pour la création d'un Etat tamoul indépendant à Sri Lanka, il semble normal que le gouvernement en place réponde de sa politique qui est déjà à l'origine de plusieurs milliers de morts. Cela était-il vraiment inévitable?

\section{Conclusion}

Les dernières attaques de l'armée ont permis une percée décisive dans les défenses des séparatistes. Plus de 60000 civils (selon $\mathrm{ONU}$ ) auraient réussi à quitter la zone de combat. Leur situation reste toutefois catastrophique pour MSF qui déplore les conditions dans lesquelles sont accueillis et soignés ces réfugiés (Nouvelobs, article du 22 avril 2009). Le rejet des différents appels à une trêve durable, émanant des Tigres ou de la communauté internationale, laisse penser que le gouvernement s'apprête à lancer l'assaut final au détriment des civils toujours piégés dans la zone de combat. Face cette catastrophe humanitaire, la communauté internationale doit continuer à faire pression pour obtenir l'arrêt immédiat des combats et pour mettre en place un couloir humanitaire afin d'évacuer les civils de la zone de combat. Il est par ailleurs inconcevable que les soi-disant 
welfare centers restent sous le contrôle de l'armée. Ces réfugiés doivent être placés sous le mandat de la communauté internationale ou pris en charge par des ONG afin de restaurer leurs droits élémentaires et d'apaiser leurs souffrances.

Il semble nécessaire de constituer un tribunal pénal international pour Sri Lanka afin d'enquêter et de poursuivre les responsables de crime de guerre et de crime contre l'humanité, aussi bien du côté des Tigres que de l'armée. Ce tribunal devra aussi déterminer si sous couvert d'une guerre contre le terrorisme, le gouvernement sri lankais ne s'est pas rendu coupable de génocide à Mullaitivu.

Il est important qu'un tribunal international statue sur cette épineuse question afin que la minorité tamoule ne se sente plus vulnérable. La perspective d'être entendus et protégés par la communauté internationale permettrait aux Tamouls de ne plus se sentir abandonnés à leur sort et ferait certainement réfléchir les dirigeants sri lankais avant d'utiliser la force, pour régler des questions politiques, au prix de milliers de morts.

\section{BIBLIOGRAPHIE}

Prakash P., Chassés avec les Tigres

http://www.liberation.fr/monde/0101318894-chasses-avec-les-tigres, consulté le 20 avril 2009

Sujeevan, Sri Lanka : Mullaithivu était-il le Srebrenica sri lankais?

http://www.tamoulobs.com/the-news/411-sri-lanka-mullaithivu-etait-il-le-srebrenica-sri-

lankais.html, consulté le 20 avril 2009

NouvelObs, MSF décrit une situation humanitaire « chaotique » au Sri Lanka, http://

tempsreel.nouvelobs.com/actualites/international/20090422.0BS4321/

msf_decrit_une_situation_humanitaire_chaotique_au_sri_l.html, consulté le 22 avril

\section{NOTES}

1. Le LTTE a été inscrit sur la liste des organisations terroristes par l'Union Européenne depuis mai 2006.

2. Tamouls originaires de la péninsule de Jaffna au nord de l'île.

\section{RÉSUMÉS}

La volonté du gouvernement sri lankais d'en finir militairement avec le LTTE a abouti à une catastrophe humanitaire. L'armée et les Tigres se rendent coupables de crime de guerre et de 
crime contre l'humanité à l'encontre des civils tamouls, qui sont piégés dans la zone de combat ou enfermés dans des camps de détention. La perception différenciée de l'opération militaire selon les communautés nécessiterait la création d'un Tribunal Pénal International pour Sri Lanka.

The will of Sri Lankan government to finish militarily with LTTE has ended with an humanitarian catastrophe. Both the Sri Lankan Army and the Tigers are guilty of international war and humanitarian crimes against Tamil civilians, who are trapped in the war zone or locked in detention camps. The different perceptions of the military operation according to the communities should need the creation of an International Penal Court for Sri Lanka

\section{INDEX}

Mots-clés : Sri lanka, guerre, tamoul, crise humanitaire, génocide

Keywords : war, tamil, humanirian crisis

\section{AUTEUR}

\section{DELON MADAVAN}

Delon Madavan(delonjb@hotmail.com) est doctorant et moniteur en géographie à l'Université Paris-Sorbonne. Il travaille actuellement, sous la direction du Professeur Olivier Sevin, sur la minorité tamoule à Colombo, Kuala Lumpur et Singapour. D. Madavan vient de passer deux mois de terrain à Sri Lanka. L'auteur a publié dans la collection Grafigeo de l'UMR PRODIG : Jaffna et le conflit intercommunautaire à Sri Lanka. 